ARTICLE

\title{
Large-Scale Simulation of Ductile Fracture Process of Microstructured Materials
}

\author{
Rong TIAN* and Chaowei WANG \\ Institute of Computing Technology, Chinese Academy of Sciences, \\ Zhongguancun Kexueyuan Nanlu 6, Haidian, Beijing 100190, PR China
}

\begin{abstract}
The promise of computational science in the extreme-scale computing era is to reduce and decompose macroscopic complexities into microscopic simplicities with the expense of high spatial and temporal resolution of computing. In materials science and engineering, the direct combination of 3D microstructure data sets and 3D large-scale simulations provides unique opportunity for the development of a comprehensive understanding of nano/microstructure-property relationships in order to systematically design materials with specific desired properties. In the paper, we present a framework simulating the ductile fracture process zone in microstructural detail. The experimentally reconstructed microstructural data set is directly embedded into a FE mesh model to improve the simulation fidelity of microstructure effects on fracture toughness. To the best of our knowledge, it is for the first time that the linking of fracture toughness to multiscale microstructures in a realistic 3D numerical model in a direct manner is accomplished.
\end{abstract}

KEYWORDS: fracture process zone, void growth, void coalescence, micro-cracking, computational science, multiscale continuum

\section{Introduction}

Material design is an iterative optimization process of finding a good match of a specific microstructure and the desired properties. So the question is: what microstructure will produce the desired material properties? To answer this question we need to clearly understand the relationship between material microstructures and the resulting properties (see Fig. 1). ${ }^{1,2)}$

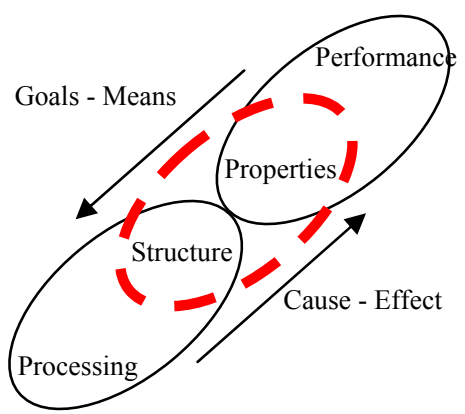

Fig. 1 "Structure-property: the missing link"1,2)

Ductile fracture occurs through void nucleation, void growth, and void coalescence. Modeling of ductile fracture has been documented in the literature in a long period of time. ${ }^{2-8)}$ Historically extensive studies have been focused on void nucleation and void growth. ${ }^{9-18)}$ Modeling of multiple void interaction and void coalescence has received far less attention in the literature. ${ }^{19)}$ Progress in void coalescence study is mainly hampered by the lack of quantitative numer-

*Corresponding author, E-mail: rongtian@ncic.ac.cn ical and experimental results to assess the validity of theoretical models; thus void coalescence remains the least understood. ${ }^{20)}$

In contrast to void growth, void coalescence is a more complex phenomenon due to several reasons: (a) Void coalescence involves strong intervoid interaction, which is historically neglected by single-void analysis; (b) Void coalescence is an unstable void growth stage; void distortion dominates this deformation stage; (c) Void coalescence poses challenges to both experimental measurements and computer simulations; (d) Knowledge of the underlying mechanisms of softening, localization and fracture in shear is more qualitative than quantitative. The mixed mode ductile fracture is still not fully understood. We refer to Fig. 2 for the micromechanics of ductile fracture, and our focus.

Recently we have successfully combined the experimentally reconstructed microstructure dataset, a multiresolution continuum theory, and large-scale parallel computing in a

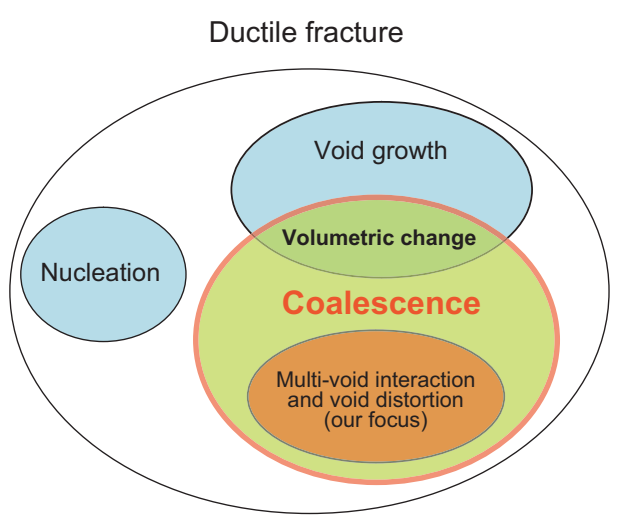

Fig. 2 Micromechanics of ductile fracture, and the recent focus

(C) 2011 Atomic Energy Society of Japan, All Rights Reserved. 


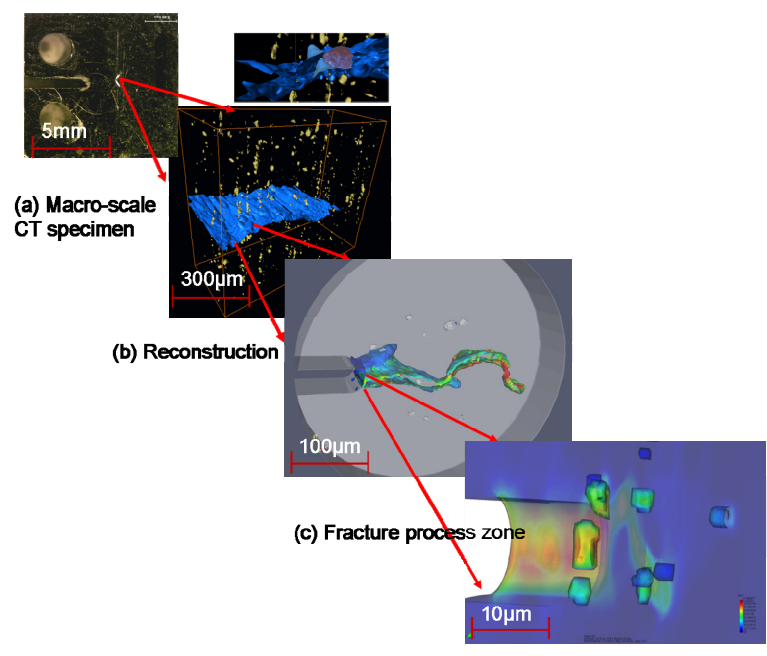

(d) Microstructural feature

Fig. 3 Three dimensional microstructure reconstructions and simulations of fracture process zone. (a) and (b) show the crack tip specimen and microstructure reconstruction providing the microstructures within the fracture process zone and crack opening displacement (COD) versus the applied load, respectively. Using high performance computing, a 3-dimensional microstructure simulation ((c), (d)) reveals clearer micro-structural features and interplay during the development of the fracture process zone and provides a deeper understanding of the effects of microstructures on materials properties.

high resolution modeling and simulation of the 3D fracture process of an ultra high strength steel. Figure 3 highlights the effort on the fracture toughness prediction directly from 3D experimentally reconstructed microstructures' kinematics. $^{21)}$

In this paper, we describe the simulation framework behind. The paper is arranged as follows. In Section II the idea, the multiscale continuum model, and the finite element implementation are introduced. In Section III numerical verifications are given and parallel performance of the developed multiscale simulation code is evaluated. In Section IV the application to the simulation of ductile fracture process zone is given, followed by the last section where conclusions are drawn.

\section{The Method}

\section{A Computational Science-Based Continuum Approach}

In the air melted high strength steel examined here, the embedded particles are generally primary particles (titanium nitrides on the order of a micron) and secondary particles (titanium carbides and manganese sulfides on the order of 10-100 nm). Hence there are two potential populations of voids, which exist at two distinct scales. As a result, we have three typical length scales to consider: macro scale, micro/primary-particle scale, sub-micro/secondary-particle scale.

DNS (direct numerical simulation) offers directly predictive capabilities from fundamental and essential principles. Nevertheless direct computational prediction of the overall behavior of materials by explicitly modeling each microscale is neither yet practical nor technically efficient. The alternative to direct simulation of a material's microstructure is material homogenization. The homogenization, however, smears out subscale material heterogeneity. As such, some important material behavior such as the inherent inhomogeneity of plastic deformation, plastic flow localization in shear bands cannot be explained. Also conventional continuum approximations cannot capture highly localized deformation fields on the order of the microstructure's characteristic length.

Motivated by these facts, we propose the following computational science based multiscale simulation approach for toughness prediction of the structural steel. A top level direct microstructure simulation is coupled to a low level of homogenization through a multiscale continuum theory. The philosophy behind is to decompose and reduce macroscopic complexities into microscopic simplicities with the expense of computing power. The idea is illustrated in Fig. 4.

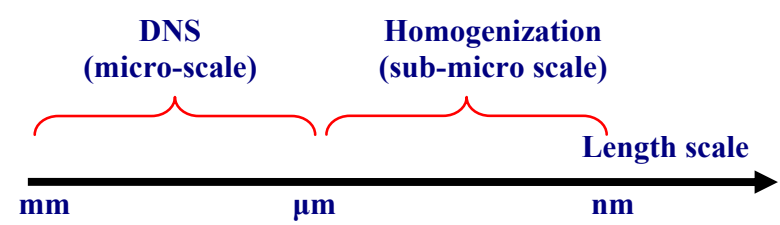

Fig. 4 Explicit microstructure multiscale simulation.

\section{Direction Numerical Simulation at Microscale}

The promise of computational science in the extreme-scale era is to reduce and decompose macroscopic complexities into microscopic simplicity with the expense of high spatial and time resolution of computing. In materials science and engineering, three dimensional explicit microstructure simulation decreases empirical input parameters and reduces phenomenological curve fitting, and so finally leads to a unique path of understanding the microstructure-property link. In particular, with advance in experimental techniques that rapidly reconstruct the three-dimensional microstructures and computational science that is at the dawn of exa-scale computing capability, three dimensional explicit microstructure simulations would give rise to new possibilities for developing a deeper understanding of the evolution of microstructures and the effects of microstructures on materials properties at an unprecedented fidelity level. Therefore, our first idea is to combine 3D microstructure data sets and 3D large-scale simulations. We envision that with petascale computing horsepower, the computational science-based simulation would provide a unique path for better understanding of microstructure-property relationships, in order to systematically design materials with specific desired properties.

\section{The Multiscale Continuum Model at Sub-Micro Scale}

Conventional continuum approximations cannot capture highly localized deformation fields on the order of the microstructure's characteristic length. And, constitutive 
behavior at these smaller scales is generally much different than the macroscale average behavior. As such, important material behavior cannot be explained, such as the inherent inhomogeneity of plastic deformation, plastic flow localization in shear bands and the effect of crack size and geometry on fracture behavior. These phenomena control the important mechanical behavior such as fracture toughness and strength. They must be captured to provide a link between structure and properties. ${ }^{1)}$

We employ the multiresolution continuum theory. ${ }^{21-29)}$ In the following formulation, $(\mathbf{D}, \boldsymbol{\sigma})$ describes the stress in the matrix material including the secondary particles. $\left(\mathbf{L}^{1}, \boldsymbol{\beta}, \overline{\boldsymbol{\beta}}\right)$ describes the inheterogeneity resulted from strain localization in the matrix. Keep in mind that the primary voids that are nucleated from the primary particles are modeled explicitly.

The generalized stress and rate of deformation for a two-scale porous metal can be expressed as

$$
\sum=\left[\begin{array}{lll}
\boldsymbol{\sigma} & \boldsymbol{\beta} & \overline{\boldsymbol{\beta}}
\end{array}\right], \quad \boldsymbol{\Delta}=\left[\begin{array}{lll}
\mathbf{D} & \left(\mathbf{L}^{1}-\mathbf{L}\right) & \mathbf{L}^{1} \bar{\nabla}
\end{array}\right]
$$

where $\boldsymbol{\sigma}$ now describes the deformation of microvoiding matrix at microscale, representing the primary void growth effect and coalescence, $\mathbf{L}^{1}$ is the local velocity gradient solved as nodal unknown, $\boldsymbol{\beta}$ describes the deformation of alloy and submicro scale, which reflects the resistance to inhomogeneous deformation at the submicro scale, the microstress couple $\overline{\boldsymbol{\beta}}$ automatically brings the length parameter into play. It is this characteristic length that makes the multiresolution continuum simulation free of the known mesh dependence issue showing up in the classical continuum simulation of strain-localization. The strong form of a two-scale continuum model is given as:

$$
\begin{array}{ll}
(\boldsymbol{\sigma}-\boldsymbol{\beta}) \cdot \bar{\nabla}+\mathbf{b}=0 & \text { in } \Omega \\
\overline{\boldsymbol{\beta}} \cdot \bar{\nabla}-\boldsymbol{\beta}=0 & \text { in } \Omega \\
(\boldsymbol{\sigma}-\boldsymbol{\beta}) \cdot \mathbf{n}=\mathbf{t} & \text { on } \Gamma_{\mathrm{t}} \\
\overline{\boldsymbol{\beta}} \cdot \mathbf{n}=0 & \text { on } \Gamma_{\mathrm{t}}
\end{array}
$$

where $\mathbf{t}$ is the traction on the surface $\Gamma_{\mathrm{t}}$ and $\mathbf{b}$ is the body force. We refer to references ${ }^{21-29)}$ for more details.

\section{Finite Element Implementation}

The 3D simulation code is developed in a generalized finite element framework in a way that can be automatically reduced back to a classical continuum code by turning off the length scale parameters. ${ }^{28-29,31)}$ This feature makes the comparison between a multiresolution continuum modeling and the classical counterpart straightforward. The central difference method is chosen to solve momentum equations because of intense local material softening and strain localization in the problem under investigation. The simulation code is written in $\mathrm{C}++$ and MPI (Message passing interface).

To gain the awards of the explicit microstructure simulation, the key is to implement the simulation code in a parallel and scalable way. The code developed has been tested and operational on PC desktops as a sequential code, and PC clusters, BlueGene, Nebulae, and Jaguar as a scalable parallel nonlinear large deformation multiscale finite element code.

\section{Benchmarks}

\section{Numerical Verification}

As we mentioned before, once the length scale parameter is disabled, the simulation code is reduced back to the standard finite element method. Using this functionality, we verify the code against $A B A Q U S^{30}$ in the context of the classical continuum simulation. A linearly hardening material is modeled by both the multiscale FEM and ABAQUS. User material subroutines UMAT and VUMAT are used to provide the customized comparison. Provided in Fig. 5(a) is the computed stress-strain relation. It is shown that msFEM, labeled for the multiscale finite element method, is in agreement with both the results of ABAQUS and the theoretical results.

In the context of the multiscale continuum, the newly developed $3 \mathrm{D}$ simulation code is verified against the $2 \mathrm{D}$ in-house code ${ }^{34)}$ in Fig. 5(b).

\section{Parallel Performance Tests}

Strong scaling test results are provided in Table 1 and Fig. 6 for "speedup", and Table 2 and Fig. 7 for "time to solution."

The tests show almost linear scalability up to 512 cores. It is worthy to mention that the finite element model at 512 cores in the benchmark contains only 241 elements (385

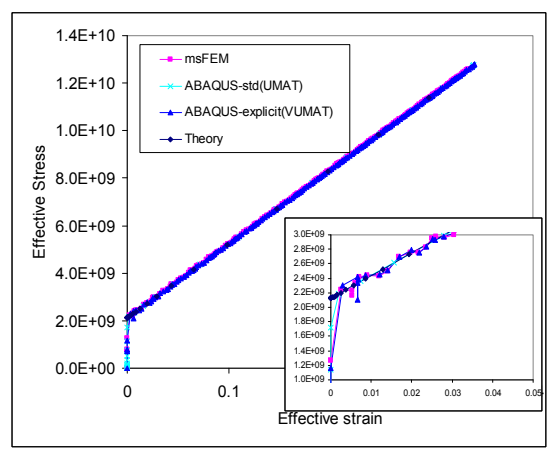

(a)

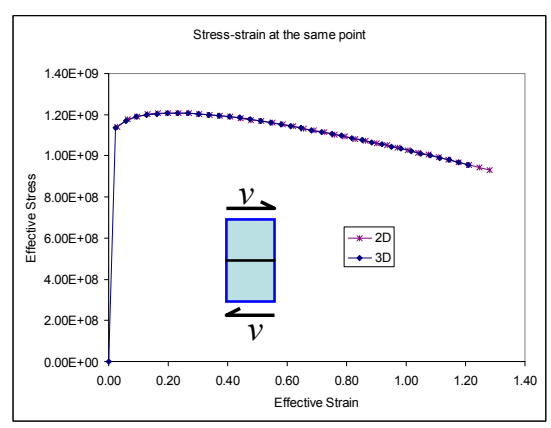

(b)

Fig. 5 Numerical verification (a) against ABAQUS, (b) with 2D multiscale analysis 
Table 1 Strong scaling test - speedup

\begin{tabular}{ccc}
\hline nProcs & Speedup & Ideal \\
1 & 1 & 1 \\
2 & 1.999 & 2 \\
4 & 3.986 & 4 \\
8 & 8.016 & 8 \\
16 & 15.608 & 16 \\
32 & 30.676 & 32 \\
64 & 61.353 & 64 \\
128 & 117.294 & 128 \\
256 & 234.588 & 256 \\
512 & 469.176 & 512 \\
\hline
\end{tabular}

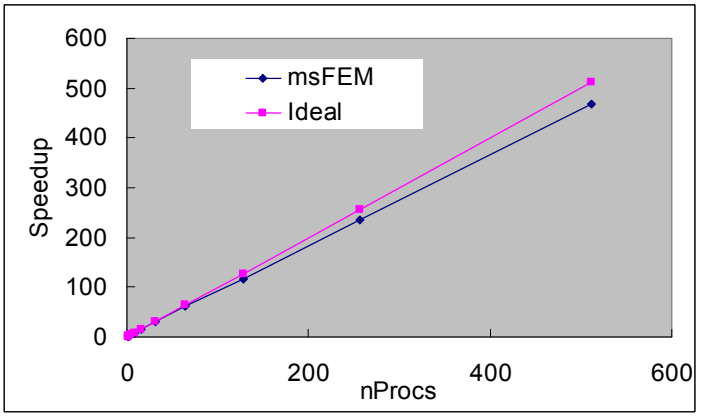

Fig. 6 Strong scaling tests -- speedup

Table 2 Strong scaling test - time to solution

\begin{tabular}{ccc}
\hline nProcs & Time to solution (s) & Ideal (s) \\
\hline 1 & 7976 & 7976 \\
2 & 3989 & 3988 \\
4 & 2001 & 1994 \\
8 & 995 & 997 \\
16 & 511 & 498.5 \\
32 & 260 & 249.3 \\
64 & 130 & 124.6 \\
128 & 68 & 62.3 \\
256 & 34 & 31.2 \\
512 & 17 & 15.6 \\
\hline
\end{tabular}

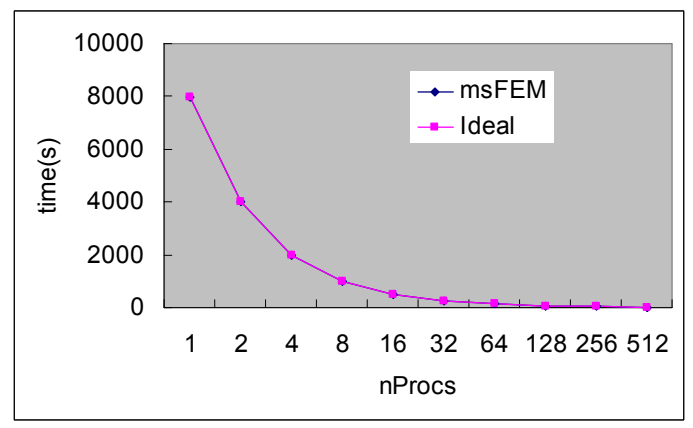

Fig. 7 Strong scaling tests -- time to solution

nodes) per single core, of which almost the half, i.e., 103 elements (119 nodes), are located in halo areas and require communications. Even so, as demonstrated in Table 2, the code still maintains a perfect speedup. The time-to-solution is shown to be as short as 17 seconds at the scale of 512 cores, only 1.4 seconds behind the ideal value, producing more than $90 \%$ parallel efficiency at scale.

Strong scaling test, with a 5 millions of elements of FE model and up to 12 thousands of cores, is provided in Fig. 8.

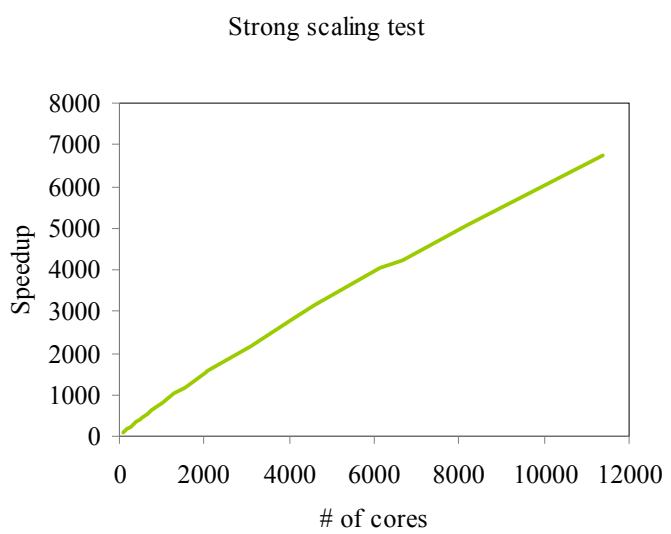

Fig. 8 Strong scaling test up to $12 \mathrm{~K}$ cores.

\section{Fracture Process Zone Simulation - How Duc- tile Fracture Develops}

As shown in Fig. 9, the creation of the numerical model starts by decomposing the domain of interest into three nested subdomains: the outer K-field, the plastic zone, and the inner process zone. The dimensions of the process zone are assumed small (several hundred $\mu \mathrm{m}$ according to the experimental reconstruction) compared to the characteristic dimensions of the K-field, and any plasticity is confined to the plastic zone. Inside the numerical process zone, the experimentally reconstructed microstructures are embedded directly. Under the small scale yielding assumption the displacement field along the outer boundary of the K-field can be calculated using the equations of linear elastic fracture mechanics (LEFM), ${ }^{33)}$

$$
\begin{aligned}
& u_{x}=\frac{K_{\mathrm{I}}}{2 \mu} \sqrt{\frac{R}{2 \pi}} \cos \left(\frac{\theta}{2}\right)\left[k-1+2 \sin ^{2}\left(\frac{\theta}{2}\right)\right] \\
& u_{y}=\frac{K_{\mathrm{I}}}{2 \mu} \sqrt{\frac{R}{2 \pi}} \sin \left(\frac{\theta}{2}\right)\left[k-1-2 \cos ^{2}\left(\frac{\theta}{2}\right)\right] \\
& u_{z}=0
\end{aligned}
$$

where $R$ and $\theta$ are the distance and the angle from the crack tip, respectively, $\mu$ is the shear modulus, $k=3-4 v$

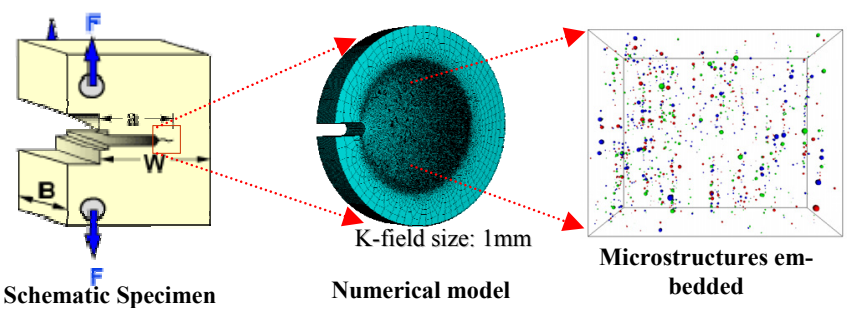

Fig. 9 The simulation framework of 3D ductile fracture process zone 


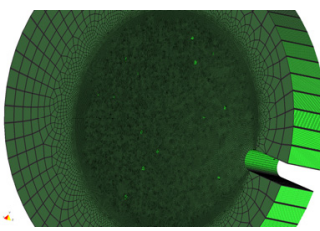

(a) Mesh

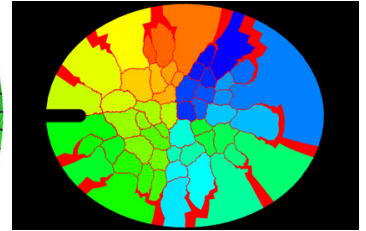

(b) Domain Decomposition
Fig. 10 Finite element mesh used in simulation

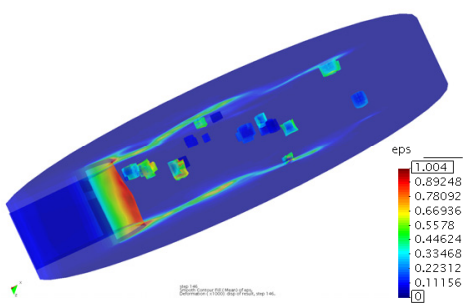

(a)

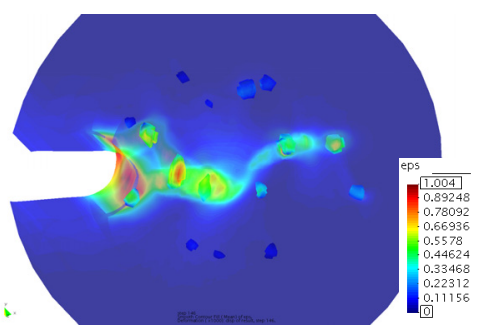

(b)

Fig. 11 Ductile process zone. (a) top view; (b) side view of ductile fracture process zone.
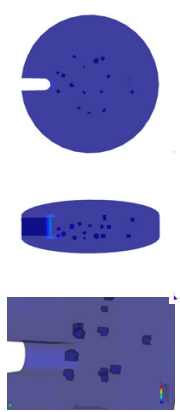

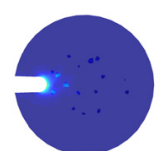

(a) Side view

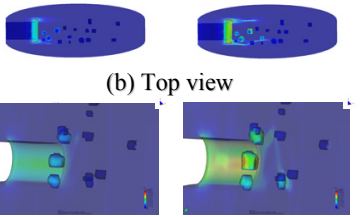

(c) Isometric view
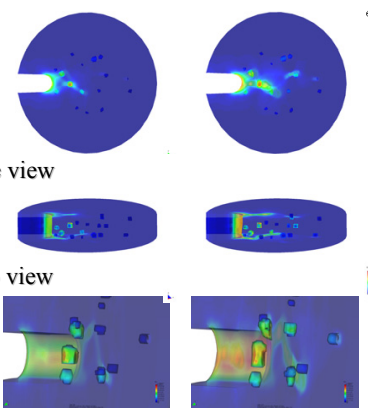

Fig. 12 Multiple void kinematics (void growth and shear coalescence) during ductile fracture process

for plane strain, $v$ is the Poisson ratio. Loading is implemented by increasing $K_{\mathrm{I}}$ to $1.5 K_{\mathrm{IC}}$. In the end, the fracture toughness $K_{\mathrm{IC}}$ is related to microstructures' (voids') kinematics.

The finite element mesh used is shown in Fig. 10(a) and the domain decomposition is employed for parallel computing (Fig. 10(b)). Figures 11(a) and (b) show the obtained ductile fracture process zone, viewed respectively from the top and the side of the specimen.

Figure 12 shows the microstructural view of ductile fracture development. The typical micromechanism of ductile fracture is revealed: plastic deformation and void growth result in crack blunting, while the coalescence and linking of
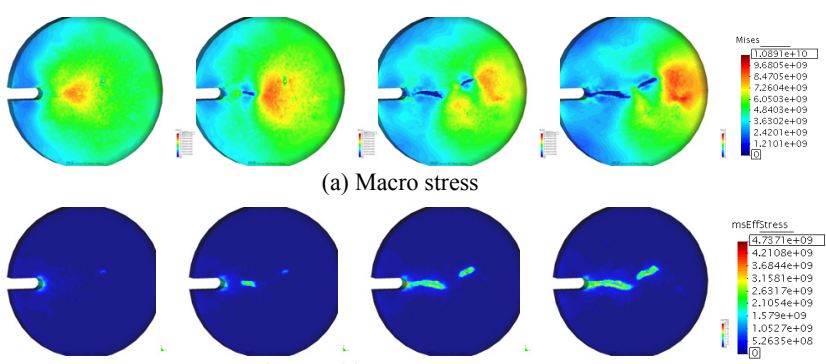

(a) Macro stress
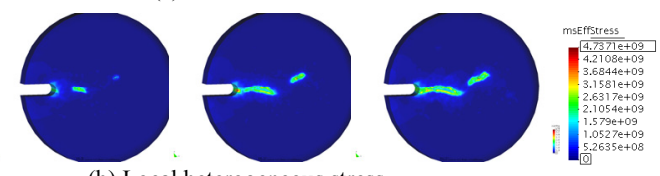

(b) Local heterogeneous stress

Fig. 13 Effective macro Stress and effective micro stress (local heterogeneous stress)

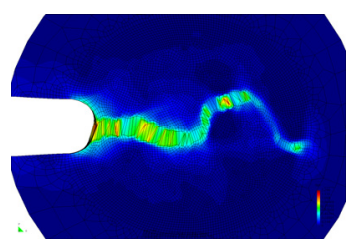

(a) Classical continuum

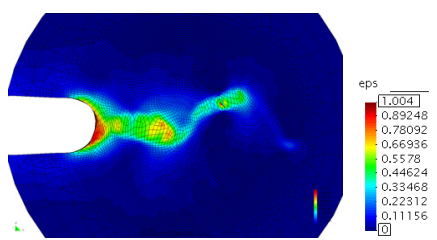

(b) Multi-scale continuum
Fig. 14 Comparison with the classical continuum simulation. The conventional continuum solution exhibits the typical spurious localization of the plastic strain in a region determined by the element size.

neighboring voids in front of the fatigue notch lead to crack advance.

Figure 13 shows the evaluation of macro stress and micro stress ahead of fatigue crack tip. The softening of the macro stress is associated with the emergence of the local heterogeneous micro-stress which triggers strain localization at submicro scale.

Finally, we repeat the process zone simulation work using the classical continuum theory and a comparison is provided in Fig. 14. One immediately observes that the classical continuum calculation (Fig. 14(a)) leads to a mesh dependent result, exhibiting the typical spurious localization of the plastic strain in a region determined by element size. This non-physical behavior has the effect of underestimating the energy release rate necessary for crack advance and therefore the fracture toughness.

\section{Conclusion}

In the short paper, we have briefly introduced a framework simulating ductile fracture process zone in microstructural detail to relate the fracture toughness property of materials to microstructures. More details including the experimental validation can be referred to the recent paper. $^{21)}$ The preliminary results show the certain potential of the simulation framework in capturing multiscale microstructures' kinematics during fracture process and the relation with the fracture toughness property. To the best of our knowledge, it is for the first time that the linking of fracture toughness of the high strength steel alloy to multiscale microstructures in a realistic large $3 \mathrm{D}$ model in a direct manner has been achieved. 


\section{Acknowledgments}

The research is financially supported by National Natural Science Foundation of China (grant No. 11072241, $11111140020,60633040)$ and the "100 Talents Program" of Chinese Academy of Sciences. Computing facilities at Beijing Supercomputing Center, and Dawning Nebulae are acknowledged. The work is partially supported by the "Director's Discretion" project of NCCS (grand \#: MAT028). Performance tests on Jaguar have been done by Yingrui WANG.

\section{References}

1) G. B. Olson, "Designing a new material world," Science, 288[5468], 993-998 (2000).

2) C. McVeigh, F. Vernerey, W. K. Liu. C. Brinson, "Multiresolution analysis for material design," Comput. Meth. Appl. Mech. Eng., 195[37-40], 5053-5076 (2006).

3) J. R. Rice, D. M. Tracey, "On the ductile enlargement of voids in triaxial stress fields," J. Mech. Phys. Solids, 17, 201-217 (1969).

4) J. R. Rice, M. L. Johnson, "The role of large crack tip geometry changes in plane strain fracture," In: M. F. Kanninen et al. (eds), Inelastic behaviour of solids, New York, McGraw-Hill, 641-672 (1970).

5) V. Tvergaard, A. Needleman, "An analysis of ductile rupture modes at a crack tip," J. Mech. Phys. Solids, 35[2], 151-183 (1987).

6) V. Tvergaard, J. W. Hutchinson, "The relation between crack growth resistance and fracture process parameters in elastic-plastic solids," J. Mech. Phys. Solids, 40[6], 1377-1397 (1992).

7) S. Hao, W. K. Liu, B. Moran, F. J. Vernerey, G. B. Olson, "Multi-scale Constitutive Model and Computational Framework for the Design of Ultra-high Strength steels," Comput. Meth. Appl. Mech. Eng., 193: 1865-1908 (2004).

8) J. S. Wang, G. B. Olson, "Prediction of Ductile Fracture Toughness," Proc. of the 12th International Fracture Conference, Ottawa, Canada, July 12-17, 2009 (2009).

9) V. Tvergaard, "Influence of voids on shear band instabilities under plane strain conditions," Int. J. Frac. Mech., 17, 389-407 (1981).

10) V. Tvergaard, A. Needleman, "Analysis of the cup-cone fracture in a round tensile bar," Acta Metallurgica, 32, 157-169 (1984).

11) A. Needleman, "A continuum model for void nucleation by inclusion debonding," J. Appl. Mech., 54, 525-531 (1987).

12) A. Needleman, J. R. Rice, "Limits to ductility set by plastic flow localization," In: D. P. Koistinen, N.-M. Wang (eds.), Mechanics of sheet metal forming, Plenum Publishing, New York, 237-267 (1978).

13) A. Needleman, V. Tvergaard, J. W. Hutchinson, "Void growth in plastic solids," In: A. S. Argon (ed.), Topics in fracture and fatigue, Springer-Verlag, New York, 145-178 (1992).

14) F. A. McClintock, S. M. Kaplan, C. A. Berg, "Ductile fracture by hole growth in shear bands," Int. J. Frac., 2[4], 614-627 (1966).

15) B. J. Lee, M. E. Mear, "Axisymmetric deformations of power-law solids containing a dilute concentration of aligned spheroidal voids," J. Mech. Phys. Solids, 40, 1805-1836
(1992).

16) M. Gologanu, J. B. Leblond, J. Devaux, "Approximate models for ductile metals containing nonspherical voids - case of axisymmetric oblate ellipsoidal cavities," J. Eng. Mater. Tech., 116, 290-297 (1994).

17) F. A. McClintock, "Local criteria for ductile fracture," Int. J. Frac., 4, 103-130 (1968).

18) P. F. Thomason, Ductile Fracture of Metals, Oxford, Pergamon Press (1990).

19) T. Pardoen, J. W. Hutchinson, "An extended model for void growth and coalescence," J. Mech. Phys. Solids, 48, 2467-2512 (2000).

20) A. Weck, D. S. Wilkinson, "Experimental investigation of void coalescence in metallic sheets containing laser drilled holes," Acta Materialia, 56, 1774-1784 (2008).

21) R. Tian, S. Chan, S. Tang, A. M. Kopacz, J.-S. Wang, H.-J. Jou, L. Siad, L.-E. Lindgren, G. Olson, W. K. Liu, "A multiresolution continuum simulation of the ductile fracture process," $J$. Mech. Phys. Solids (2010) [DOI: 10.1016/j.jmps.2010.07.002]

22) R. Tian, A. C. To, W. K. Liu. "Conforming local meshfree method," Int. J. Numer. Meth. Eng., 86[3], 335-357 (2011).

23) H. Kadowaki, W. K. Liu, "A multiscale approach for the micropolar continuum model," Comput. Model. Eng. Sci., 7[3], 269-282 (2005).

24) F. J. Vernerey, C. McVeigh, W. K. Liu, B. Moran, D. Tewari, D. M. Parks, G. B. Olson, "The 3-D computational modeling of shear-dominated ductile failure in steel," J. Miner. Met. Mater. Soc., 58, 45-51 (2006).

25) F. J. Vernerey, W. K. Liu, B. Moran, "Multi-Scale Micromorphic Theory for Hierarchical Materials," J. Mech. Phys. Solid, 55[12], 2603-2651 (2007).

26) F. J. Vernerey, W. K. Liu, B. Moran, G. B. Olson, “A micromorphic model for the multiple scale failure of heterogeneous materials," J. Mech. Phys. Solids, 56[4], 1320-1347 (2008).

27) C. McVeigh, W. K. Liu, "Linking microstructure and properties through a predictive multiresolution continuum," Comput. Meth. Appl. Mech. Eng., 197, 3268-3290 (2008).

28) C. McVeigh, W. K. Liu, "Multiresolution modeling of ductile reinforced brittle composites," J. Mech. Phys. Solids, 57, 244-267 (2009).

29) R. Tian, B. Moran, W. K. Liu, G. B. Olson, Multiscale fracture simulator, Dynamic Microstructure Design Consortium (ONR Contract: N00014-05-C-0241) Base Final Report (2008).

30) R. Tian, W. K. Liu, S. Chan, G. B. Olson, S. Tang, J. S. Wang, H. J. Jou, J. D. Gong, B. Moran, Linking Microstructures to Fracture Toughness -- predictive $3 D$ process zone simulations, The D 3-D Annual PI Review, Evanston, IL, March 23-25 (2009)

31) http://www.simulia.com/

32) R. Tian, G. Yagawa, "Non-matching mesh gluing by meshless interpolation - an alternative to Lagrange multipliers," Int. J. Numer. Meth. Eng., 71, 473-503 (2007).

$33)$ W. K. Liu, L. Siad, R. Tian, S. Lee, D. Lee, X. Yin, W. Chen, S. Chan, G. B. Olson, L.-E. Lindgren, M. F. Horstemeyer, Y.-S. Chang, J.-B. Choi, Y. J. Kim, "Complexity Science of Multiscale Material via Stochastic Computations," Int. J. Numer. Meth. Eng. (2009) [DOI: 10.1002/nme.2578].

34) M. F. Kanninen, C. H. Popelar, Advanced Fracture Mechanics, John Wiley and Sons, Oxford (1985).

35) C. McVeigh, PhD thesis, Northwestern University (2007). 